\title{
Dominance of ipsilateral corticospinal pathway in congenital mirror movements
}

\author{
Y Ueki, T Mima, T Oga, A lkeda, T Hitomi, H Fukuyama, T Nagamine, H Shibasaki
}

J Neurol Neurosurg Psychiatry 2005;76:276-279. doi: 10.1136/jnnp.2004.040949

\begin{abstract}
Objective: To clarify the mechanism of congenital mirror movements.

Design: The triple stimulation technique (TST) and the silent period were used to investigate a patient with congenital mirror movements. The TST was used to calculate the ratio of ipsilateral to contralateral corticospinal tracts from the two hemispheres to the spinal motor neurones.

Results: Transcranial magnetic stimulation over unilateral MI induced larger ipsilateral than contralateral motor evoked potentials on both sides. Only $9 \%$ of spinal motor neurones innervating the abductor digitorum minimi were excited by contralateral primary motor cortex (M1) stimulation, while $94 \%$ were excited by the ipsilateral M1 stimulation. The silent period was examined during mirror movements and with voluntary contraction of the right first dorsal interosseus mimicking mirror movements. Left MI stimulation (through the crossed corticospinal tract) did not show any difference in silent period between the two conditions, while right $\mathrm{MI}$ stimulation (through the uncrossed tract) caused a longer silent period during mirror movements than during voluntary contractions.

Conclusions: The results suggest that mirror movements may be caused by a strong connection between ipsilateral M1 and the mirror movements conveyed through a dominant ipsilateral corticospinal pathway.
\end{abstract}

M irror movement is a synkinesis occurring in the opposite hand during an intended hand movement and is characterised by simultaneous contraction of homologous muscles in both hands. Although mirror movement of moderate degree is seen physiologically in early childhood, the persistence of conspicuous mirror movements in adults has been associated with pathological conditions. ${ }^{1}{ }^{2}$ Mirror movements can be caused by unintended bilateral Ml activation because of the lack of transcallosal inhibition; they may also be caused by unilateral Ml activation resulting in bilateral movements through an abnormal projection of the corticospinal tract, either ipsilateral to or contralateral to the intended movement. $^{3-6}$ So far, however, quantitative functional analysis of the corticospinal tract in this condition has not been reported.

The triple stimulation technique (TST), described by Magistris et al in $1998,{ }^{7}$ is based on a principle of double collisions of impulses elicited by peripheral stimulation, combined with transcranial magnetic stimulation (TMS). By suppressing the desynchronisation of motor evoked potentials (MEP), this technique enables us to quantify the contribution of the cortical-motor neurone pool to the target muscle.

In the present study, we applied this technique to calculate the ratio of ipsilateral to contralateral corticospinal projec- tion, and investigated its pathophysiological role in a patient with congenital mirror movements.

\section{METHODS}

The patient was a 20 year old right handed man, who had suffered from mirror movements affecting his hands and forearms bilaterally since childhood, without any progression (grade 3 in the right hand, grade 2 in the left hand, according to the criteria of Woods and Teuber ${ }^{8}$ ). His mother was also noted to have mirror movements. General physical examination was normal, including olfaction. When he intended to grasp with one hand, the grasping powers of the right and left hand were 19 and $17 \mathrm{~kg}$, respectively, whereas when he intended to grasp with both hands simultaneously, grasping power increased to $35 \mathrm{~kg}$ for each hand (average of 10 trials for each). Magnetic resonance imaging of the cervical spine and head showed no abnormalities.

In order to monitor hand movement, surface electromyograms (EMGs) were recorded from the first dorsal interosseus (FDI) and the abductor digitorum minimi (ADM) muscles bilaterally from a pair of $\mathrm{Ag}-\mathrm{AgCl}$ electrodes (bandpass filter, 5-500 Hz; A/D rate, $2000 \mathrm{~Hz}$; Neuroscan Co, Herndon, Virginia, USA).

Focal TMS was delivered by a Magstim 200 stimulator (Magstim Co, Whitland, Dyfed, UK), using a figure of eight shaped coil (external diameter, $7 \mathrm{~cm}$ ). The centre of the coil was situated slightly laterally, towards the stimulated hemisphere. Slight displacements were made in all directions until the position producing the maximum contralateral and ipsilateral ADM responses was found. The amplitude and latency of the MEPs were measured in the relaxed condition.

In order to calculate the ratio of ipsilateral to contralateral corticospinal pathways, we used TST, as reported by Magistris et al. ${ }^{7}$ TST was started with measurement of the compound muscle action potential (CMAP) and its latency evoked by supramaximal electrical stimulation of the right ulnar nerve at the right wrist and at right Erb's point with both ADM muscles under resting conditions (fig lA). The MEPs were obtained from the right ADM using magnetic transcranial stimuli over either Ml. Three stimuli were given, leading to two collisions (fig 1C). First, the supramaximal cortical stimulation ( $99 \%$ of the machine output) was applied to the left or right $\mathrm{Ml}$ (fig 1B). After a delay of $18 \mathrm{~ms}$ (latency difference between the TMS evoked MEP and $\mathrm{CMAP}_{\text {wrist, }}$ $\mathrm{l} \mathrm{ms}$ ), the right ulnar nerve was stimulated by a supramaximal electrical pulse at the wrist, which is expected to produce the collision with the above descending impulse. Eight milliseconds after the ulnar nerve stimulation (latency difference between the $\mathrm{CMAP}_{\text {Erb }}$ and $\mathrm{CMAP}_{\text {wrist, }} \mathrm{lms}$ ), a supramaximal electrical pulse was delivered to the right Erb's

Abbreviations: ADM, abductor digitorum minimi; CMAP, compound muscle action potential; FDI, first dorsal interosseus; MEP, motor evoked potentials; TMS, transcranial magnetic stimulation; TST, triple stimulation technique 
A MEP

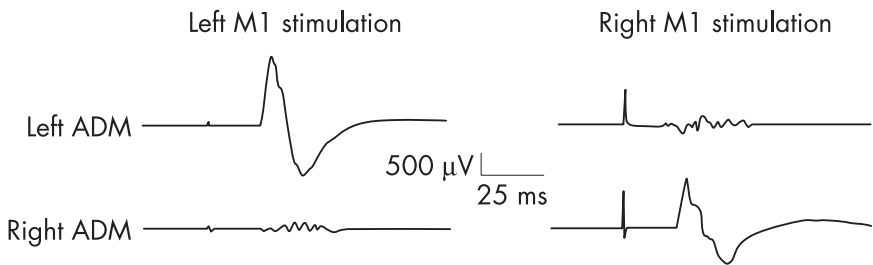

B TST

(recorded from right ADM)
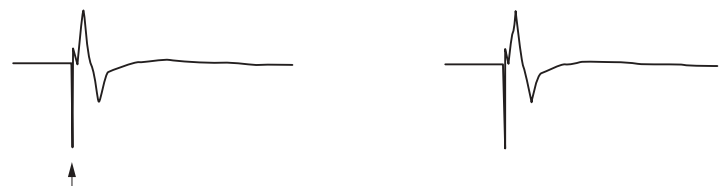

Wrist stimulation
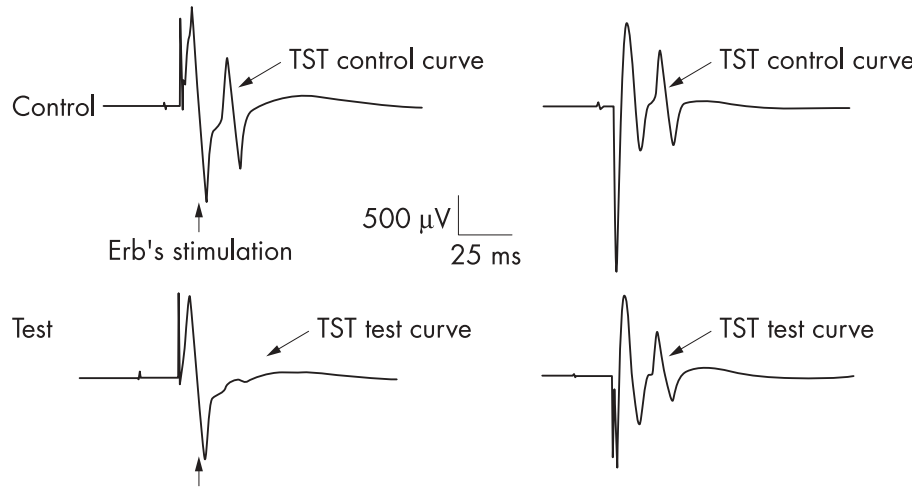

Erb's stimulation

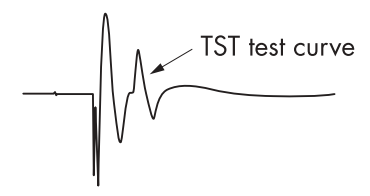

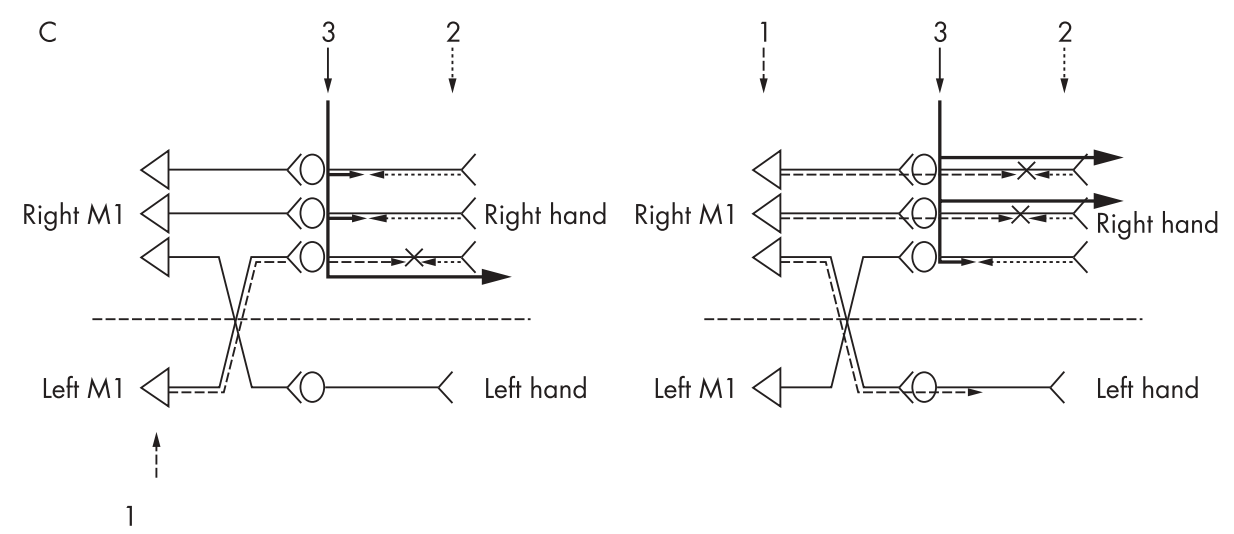

point, which is expected to cause another collision with the ascending impulse produced by the wrist stimulation, depending on the degree of the first collision. Thus the final motor response reflects the number of the motor neurones innervated by contralateral or ipsilateral Ml to the right hand muscles. As a test for the TST study, a triple stimulation set was carried out by successive stimulation of the left or right M1, the right wrist, and right Erb's point (TMS-wrist-Erb) after appropriate adjustment of the delays to give two collisions. As a control for the TST study, another triple stimulation set was carried out by successive stimulation of Erb's point, wrist, and Erb's point on the right (Erb-wristErb), with appropriate adjustments of the delays $(8 \mathrm{~ms})$. The amplitude ratio of MEPs elicited by TST $v$ the control study was used to evaluate the proportion of spinal motor neurones activated by TMS. Although we tried to carry out TST
Figure 1 (A) Results of conventional triple stimulation technique (TST) study. Focal transcranial magnetic stimulation (TMS) over the left or right $M 1$ caused motor evoked potentials (MEPs) of normal latency in the abductor digitorum minimi ( $A D M$ ) muscles contralaterally and ipsilaterally under resting conditions. Whether the left or the right $M 1$ was stimulated, the ipsilateral MEP was larger than the contralateral MEP. Note that the MEP is small and polyphasic. TMS intensity was set at $68 \%$ of the machine output for both sides. (B) Results of TST study. See the text for full description. (C) Schematic diagram explaining the possible pathogenesis of mirror movements in this patient. The schematic drawing represents the expected effect of TST over either $\mathrm{MI}$ to the right hand muscles. Arabic numbers refer to the successive TST stimulations. Broken lines show the pyramidal tract excited by the initial supramaximal magnetic $\mathrm{M} 1$ stimulation. After a delay, a second stimulus (dotted lines) applied to the right wrist evokes a collision with the descending impulse (crosses). After another delay, a third stimulus (thick lines) applied to the right Erb's point causes another collision with the ascending impulse from the second stimulation. The final motor responses reflect the number of motor neurones innervated by contralateral or ipsilateral $M 1$ to the right hand muscles (left and right panel, respectively). Thus this patient with mirror movements has a dominant ipsilateral corticospinal pathway. recorded from the left ADM, we could not achieve supramaximal electrical stimulation at the right Erb's point.

We defined the silent period as a period of EMG silence following TMS delivered over either Ml during the right FDI muscle contraction. The silent period was measured from the right FDI muscles under two conditions-while the right FDI muscle was contracting because of mirror movements during $50 \%$ maximal volitional contraction of the left FDI; and while the patient was volitionally contracting the right FDI to the same degree as the EMG level of mirror movements. As the EMG level affects the duration of the silent period, care was taken to ensure that the patient could match the volitional contraction and mirror movements with the aid of EMG visual feedback. We measured silent period duration from the stimulus to the beginning of the post-MEP EMG activity on the single trial rectified and averaged EMG. To measure the 
Table 1 Silent periods during mirror movements $v$ voluntary contraction mimicking mirror movements

\begin{tabular}{llll}
\hline Pyramidal tract carrying stimuli & $\begin{array}{l}\text { Stimulated } \\
\text { hemisphere }\end{array}$ & $\begin{array}{l}\text { Condition of the target } \\
\text { muscle }\end{array}$ & Silent period (ms) \\
\hline Uncrossed corticospinal tract & $\begin{array}{l}\text { Right } \\
\text { Right }\end{array}$ & $\begin{array}{l}\text { Mirror movements } \\
\text { Voluntary contraction }\end{array}$ & $\begin{array}{l}158.4(4.25)^{*} \\
141.9(5.24)\end{array}$ \\
Crossed corticospinal tract & Left & $\begin{array}{l}\text { Mirror movements } \\
\text { Voluntary contraction }\end{array}$ & $145.8(4.81)$ \\
& Left & $142.9(6.38)$ \\
\hline
\end{tabular}

Values are mean (SD), recorded from right first dorsal interosseus.

${ }^{*} \mathrm{p}<0.05$.

silent period, 10 trials were recorded for each condition and the mean value adopted.

The patient gave his written informed consent to the study, which was approved by ethics committee of Kyoto University School of Medicine.

\section{RESULTS}

Focal TMS over either the left or the right Ml evoked MEPs in the ADM muscles both contralaterally and ipsilaterally under resting conditions, and their onset latencies were 20.4 ms contralaterally and $20.8 \mathrm{~ms}$ ipsilaterally for both sides. The coil handles pointed backward and $45^{\circ}$ away from the midline. The optimal positions for the ipsilateral and contralateral ADM were not significantly different. Whether the left or the right Ml was stimulated, the ipsilateral MEP was larger than the contralateral MEP, which was very small and polyphasic (when stimulating the left $\mathrm{Ml}$, the MEP amplitude in ADM was $3.05 \mathrm{mV}$ on the left and $0.23 \mathrm{mV}$ on the right; when stimulating the right $\mathrm{Ml}$, it was $0.35 \mathrm{mV}$ on the left and $2.30 \mathrm{mV}$ on the right) (fig lA).

In the TST study (TMS-wrist-Erb) with TMS applied to the left $\mathrm{Ml}$, the amplitude ratio of the right ADM against the control study (Erb-wrist-Erb) was 9\%. In contrast, when the TMS was applied to the right Ml and MEP was recorded from the right ADM, the corresponding amplitude ratio was $94 \%$ (fig lB). In both cases, the machine output was supramaximal $(99 \%)$, so that the increase in TMS strength did not affect the MEP sizes.

In the silent period study applying TMS to the right $\mathrm{Ml}$ with the intensity of $120 \%$ of the resting motor threshold ( $68 \%$ of the machine output), the silent period induced in the right FDI during mirror movements of the right FDI was significantly longer than that during voluntary contraction of the right FDI mimicking mirror movements (mean (SD): 158.4 (4.25) ms for mirror movements $v 141.9$ (5.24) $\mathrm{ms}$ for voluntary contraction; $\mathrm{p}<0.05$ by $t$ test). In contrast, the silent period induced in the right FDI by TMS of the left Ml was not significantly different between mirror movements of the right FDI and voluntary contraction of the right FDI mimicking mirror movements (145.8 (4.81) ms for mirror movements $v 142.9$ (6.38) ms for voluntary contraction) (table 1).

\section{DISCUSSION}

This study provides the first quantitative evidence of abundant uncrossed corticospinal innervation in mirror movements. In this patient, focal TMS over unilateral Ml induced MEPs in bilateral hand muscles with equally normal latency, suggesting the presence of fast conducting corticospinal tracts directly connecting $\mathrm{Ml}$ to bilateral spinal motor neurones. ${ }^{2} 9$ In addition, whether the left or the right Ml was stimulated, the ipsilateral MEP was much larger than the contralateral MEP for both sides.

In X linked Kallmann's syndrome, the apparent ratio of the ipsilateral to the contralateral MEP size showed considerable variation across subjects with respect to the degree of decussation of the corticospinal tract at the level of the pyramid. ${ }^{9}$ Recently, coherence and cumulant analysis between EEG and EMG in patients with mirror movements demonstrated that the variable size ratio of the ipsilateral to the contralateral MEP was relevant to the varying degrees of ipsilateral $v$ contralateral drive that result in mirror movements. ${ }^{10}$ However, quantitative analysis of MEP amplitude is not straightforward because MEP is smaller than peripherally induced CMAP, mostly because of the desynchronisation of the TMS induced spinal motor neuronal discharges. TST can overcome this drawback. In normal human subjects, TMS over Ml activates more than $90 \%$ of the contralateral spinal motor neurones. ${ }^{7}$ In the present case, the right Ml stimulation activated 94\% of the ipsilateral spinal motor neurones innervating the right hand muscle, suggesting that the corticospinal tract is mostly derived from the ipsilateral Ml. This provides evidence that the aberrant pattern of ipsilateral $v$ contralateral corticospinal pathways is involved in producing mirror movements in the present patient. Although recrossing again at the spinal levels after the original pyramidal crossing may be another possibility, it is likely that the dominant ipsilateral corticospinal pathway may have resulted from a lack of pyramidal decussation.

When the present patient made simultaneous grasps with both hands, the grasping power increased to $194 \%$ in each hand compared with the unilateral grasp. In normal subjects, the maximum force levels produced during simultaneous bilateral contractions are almost $10 \%$ lower than those during unilateral contractions, the phenomenon known as "bilateral strength deficit". ${ }^{11}$ To our knowledge, this paradoxical "bilateral strength increase" in a mirror movement patient has not been described before, and it might be associated with a synergistic effect from both Ml to each hand.

To investigate the generator mechanism of mirror movements, silent periods were examined during mirror movements and voluntary contractions mimicking mirror movements. Silent periods generated by the stimulation of the crossed corticospinal tract did not show any difference between the two conditions, whereas those generated by the uncrossed tract were longer during mirror movements than during voluntary muscle contraction. In a previous study of mirror movement patients, the silent period during intended unilateral hand muscle contraction was abnormally short regardless of the side of Ml stimulation, which may reflect the abnormal output from the non-stimulated Ml. ${ }^{5}$ In contrast, the present case showed lengthening of the silent period during mirror movements only when TMS was applied to the uncrossed corticospinal tract. The ipsilateral silent period could either be due to the ipsilateral pathways or to transcallosal effects between the two motor cortices. ${ }^{12}$ In this case transcallosal inhibition was judged to be normal because the conditioning of TMS evoked test MEPs in the relaxed right FDI resulted in a maximum inhibition of $24.2 \%$ at 
interstimulus intervals of $10 \mathrm{~ms} \cdot{ }^{13}$ It is therefore likely that the difference in silent period duration is associated with the different degree of involvement of the ipsilateral pathway. The longer ipsilateral silent period during mirror movements may reflect the greater ipsilateral corticospinal connection during mirror movements as compared with voluntary contractions. A schematic diagram explaining the TST with its special relation to the present study of mirror movements and aberrant corticospinal pathways is shown in fig 1C. This strong connection from ipsilateral Ml to the mirror movements conveyed through the structurally dominant ipsilateral corticospinal pathway may explain the pathogenesis of mirror movements in this patient. As it is possible that the generator mechanism of mirror movements may be diverse across patients, further research in this rare disorder is necessary.

\section{Authors' affiliations}

Y Ueki, T Mima, T Oga, H Fukuyama, T Nagamine, H Shibasaki*, Human Brain Research Centre, Kyoto University Graduate School of Medicine and Faculty of Medicine, Kyoto, Japan

A lkeda, T Hitomi, H Shibasaki, Department of Neurology, Kyoto University Graduate School of Medicine and Faculty of Medicine T Oga, Helen Wills Neuroscience Institute, University of California, Berkeley, California, USA

*Current address: NINDS, National Institutes of Health, Bethesda, Maryland, USA

Competing interests: none declared

Correspondence to: Dr Tatsuya Mima, Human Brain Research Centre, Kyoto University Graduate School of Medicine, 54 Kawahara-cho, Shogoin, Sakyo-ku, Kyoto 606-8507, Japan; mima@kuhp.kyoto-u.ac.jp
Received 4 March 2004

In revised form 13 April 2004

Accepted 25 April 2004

\section{REFERENCES}

1 Farmer SF, Ingram DA, Stephens JA. Mirror movements studied in a patient with Klippel-Feil syndrome. J Physiol (Lond) 1990;28:467-84.

2 Danek A, Heye B, Schroedter R. Cortically evoked motor responses in patients with Xp22.3-linked Kallmann's syndrome and in female gene carriers. Ann Neurol 1992;31:299-304.

3 Shibasaki H, Nagae K. Mirror movement: application of movement-related cortical potentials. Ann Neurol 1983;15:299-302.

4 Cohen LG, Meer J, Tarkka I, et al. Congenital mirror movement: abnormal organization of motor pathways in two patients. Brain 1991;114:381-403.

5 Cincotta $M$, Borgheresi A, Boffi P, et al. Bilateral motor cortex output with intended unimanual contraction in congenital mirror movements. Neurology 2002;58:1290-3.

6 Gunderson CH, Solitare GB. Mirror movements in patients with the Klippel-Feil syndrome. Arch Neurol 1958;18:675-9.

7 Magistris MR, Rosler KM, Truffert A, et al. Transcranial stimulation excites virtually all motor neurons supplying the target muscle. A demonstration and a method improving the study of motor evoked potentials. Brain 1998;121:437-50.

8 Woods BT, Teuber HL. Mirror movements after childhood hemiparesis. Neurology 1978;28:1152-7.

9 Mayston MJ, Harrison LM, Quinton R, et al. Mirror movement in X-linked Kallmann's syndrome. A neurophysiological study. Brain 1997; 120:1199-216.

10 Farmer SF, Harrison LM, Mayston MJ, et al. Abnormal cortex-muscle interactions in subjects with X-linked Kallmann's syndrome and mirror movement. Brain 2004; 127:385-97.

11 Oda S, Moritani T. Maximal isometric force and neural activity during bilateral and unilateral elbow flexion in humans. Eur J Appl Physiol 1994;69:240-3.

12 Wassermann EM, Fuhr P, Cohen LG, et al. Effects of transcranial magnetic stimulation on ipsilateral muscles. Neurology 1991;41:1795-9.

13 Gerloff C, Cohen LG, Floeter MK, et al. Inhibitory influence of the ipsilateral motor cortex on stimulation of the human cortex and pyramidal tract. J Physiol (Lond) 1998;510:249-59. 